\title{
Appearance of rare physics phenomena in the OPERA neutrino experiment
}

M. Roda*i

Padova University and Padova INFN

E-mail: marco.roda@pd.infn.it

Since their discovery in 1998, neutrino oscillations are the only established phenomenon that occur beyond the standard model. So far, oscillations are also our more effective tool for the investigation of the lepton sector.

In this context, OPERA is the only experiment which is sensitive to all three neutrino flavour in the $10 \mathrm{GeV}$ region. Up to now, OPERA has discovered $4 v_{\tau}$ interaction candidates, with an overall significance of $4.2 \sigma$.

Recently, a new interesting event has been discovered with two secondary vertexes. Its preliminary analysis will be illustrated and discussed.

XVI International Workshop on Neutrino Telescopes,

2-6 March 2015

Palazzo Franchetti - Istituto Veneto, Venice, Italy

* Speaker.

†n behalf of the OPERA collaboration 


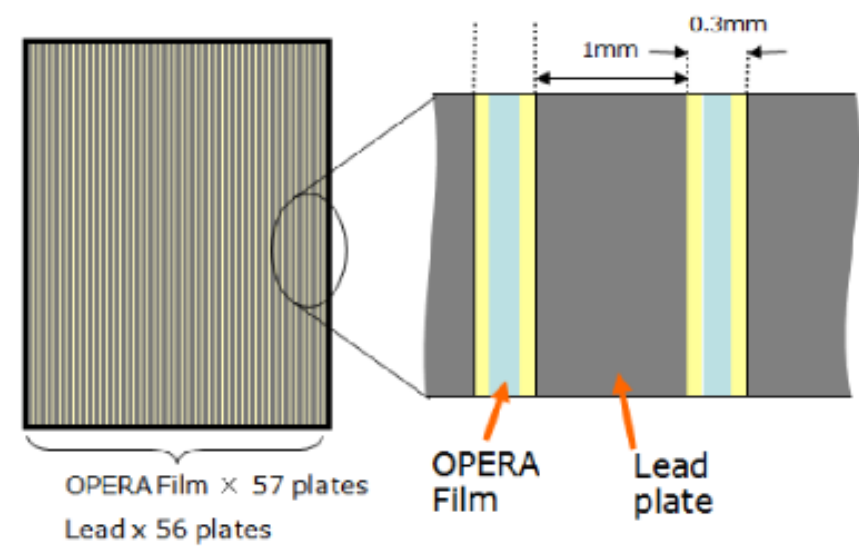

Figure 1: The OPERA target brick element is made of a succession of layers of lead and nuclear emulsions. In the transverse plane the size of the layers is $10.0 \mathrm{~cm} \times 12.5 \mathrm{~cm}$. The mass of each brick is $8.3 \mathrm{~kg}$.

\section{The OPERA experiment}

OPERA [1] is an experiment dedicated to the study of neutrino oscillation in the channel $v_{\mu} \rightarrow v_{\tau}$. In particular, the experiment is designed to observe the $v_{\tau}$ appearance on an event by event basis.

The neutrino source is the $\mathrm{CNGS}^{1}$ neutrino beam at CERN, whose energy spectrum has been optimized to maximize the rate of $v_{\tau}$ Charged Current interactions. The baseline is $730 \mathrm{~km}$, so that the probability $P\left(v_{\mu} \rightarrow v_{\tau}\right)$ is of the order of $1 \%$.

The detector concept is based on the nuclear emulsion technology, in order to achieve the spatial resolution required to identify the short tau decay track length, which is of the order of a few tenths of mm. To guarantee the modularity of the whole detector, the main active unit of the detector consists of a brick made of 56 layers of lead and nuclear emulsions, see Figure 1. The final experimental setup consists of 150 thousand bricks, disposed together with scintillators and gas detectors, see Figure 2. The latter electronic detectors are used to identify the bricks containing the primary interactions and to study the properties of the muons produced in the physics processes.

So far, $4 v_{\tau}$ neutrino interaction candidates have been reported [2] which corresponds for the observation of the $v_{\mu} \rightarrow v_{\tau}$ oscillation to a significance of $4.2 \sigma$ with respect to the background only hypothesis.

\section{A new interesting event}

Among the OPERA 2011 data sample a new interesting event has been found. The topology is singular and it is compatible with the decay of two different short-lived particles, both produced at the primary vertex. The event also presents an electromagnetic shower coming from a high energy photon. A scheme of the visible tracks can be found in Figure 3. In the electronic detectors, the

\footnotetext{
${ }^{1}$ CERN Neutrino to Gran Sasso
} 


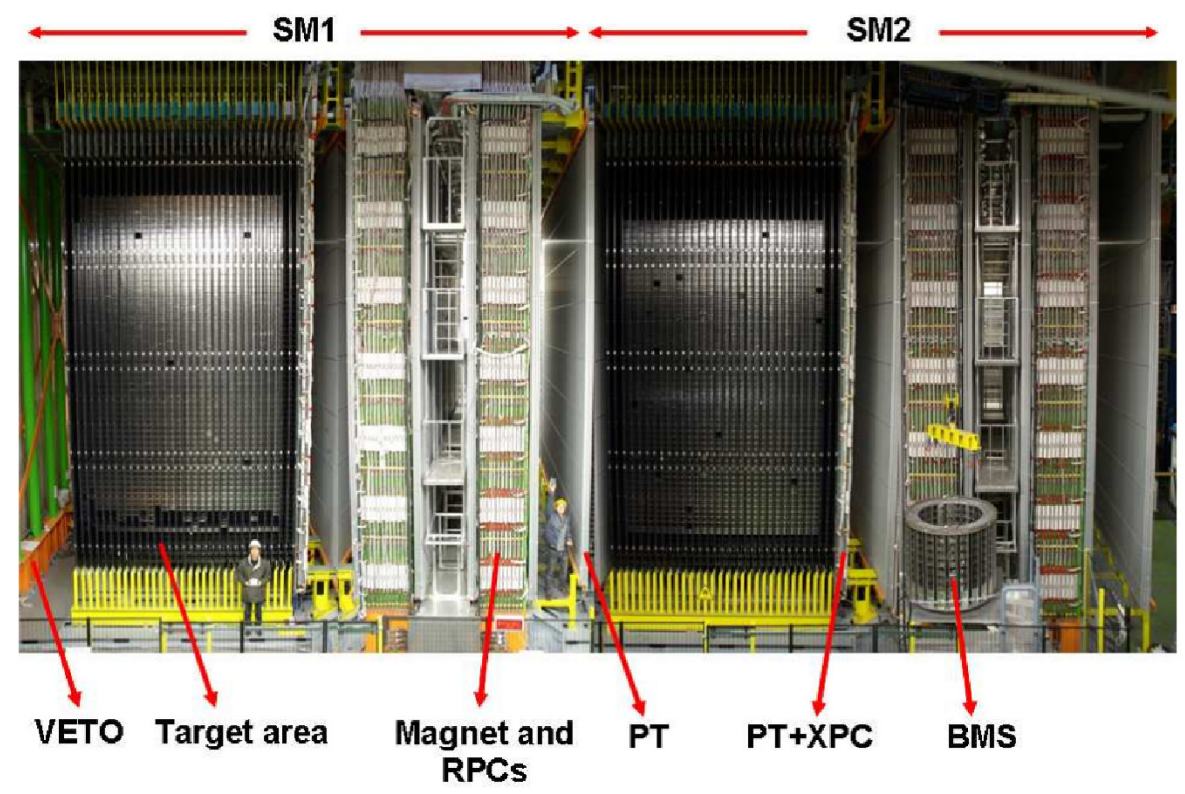

Figure 2: View of the OPERA detector which consists of two identical Super Modules. The bricks are disposed in the target areas. The neutrino beam is coming from the left.

event looks like a blob and there is no evidence for the presence of a muon, see Figure 4. The total visible energy is about $20 \mathrm{GeV}$.

The first decay is a 1-prong decay, which also presents a high energy photon clearly attached to the kink point. Preliminary estimation of the charged daughter momentum is about $3 \mathrm{GeV}$. The distance of this kink vertex from the primary vertex is $1.16 \mathrm{~mm}$, and the primary track is visible at plate 32 .

The second is more likely a 2-prong decay, but the possibility of a 3 prong is not negligible, due to a near track which could be connected to this secondary vertex instead of the primary one. The two main tracks coming out from this decay are high energy particles with a momentum estimation close to 2 and $4 \mathrm{GeV}$ respectively. The latter re-interacts in plate 53, so that it is clearly an hadron. The distance between the primary vertex and this secondary vertex is $0.103 \mathrm{~mm}$.

This peculiar and rare topology has been confirmed by two independent measurements of the emulsion films.

\section{Dedicated analysis}

This particular topology was not taken into account in the experimental proposal, but OPERA already has a criterion to select $\tau$ particles, which is very strict in order to avoid the background. The 1-prong particle is almost $\tau$-like, but it can not completely fulfill this selection criteria. So, using the standard OPERA criteria, this event can not be classified. At the same time, the 2-prong particle is very charm-like, but there is no muon at the primary vertex. This leaves the interpretation of this event quite unsatisfactory, for being such a rare observation. 


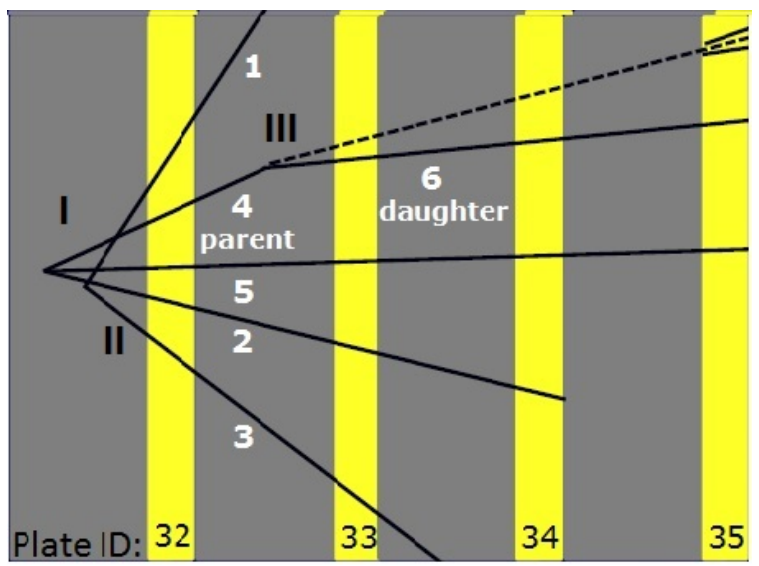

Figure 3: Scheme of the tracks reconstructed for the interesting event 11143018505 . The yellow bands correspond to the position of the emulsion layer. The dotted line is the trajectory of the photon that converts into an electron pair at plate 35 .

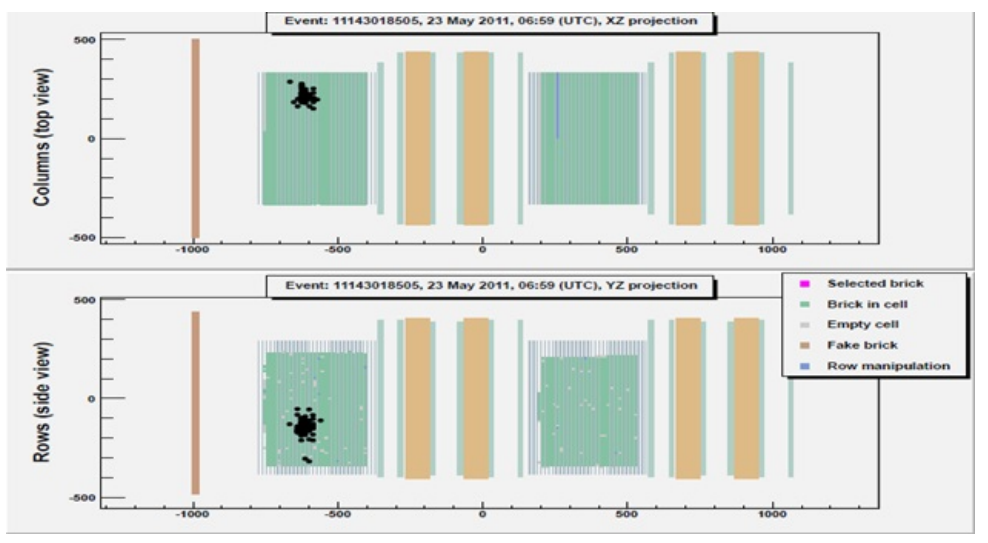

Figure 4: Event display of the interesting event. No muons are reconstructed. The total visible energy is about $20 \mathrm{GeV}$.

Anyway, the presence of two secondary vertexes in the same event is a strict selection by itself, and this can justify a looser selection criteria for the track properties which will be refined by a multivariate analysis after the topological selection.

The possible interpretations of the events are:

- $v_{\tau}$ charged current interaction with charm production, which is a process never observed so far;

- $v_{\tau}$ charged current interaction with an hadron re-interaction in the lead;

- neutrino neutral current interaction with a charm pair production, which is a rare process already observed in the CHORUS experiment [3];

- neutrino neutral current interaction with two hadron re-interactions in the lead; 
- $v_{\mu}$ charged current interaction with charm production, one hadron re-interaction and one mis-identified muon;

- $v_{\mu}$ charged current interaction production, with two hadron re-interactions and one misidentified muon;

each of them being a very rare process.

The strategy developed for the simulation is to propagate a huge amount of events in a fast simulation chain based on the GEANT4 software [4]. The input of these simulations is produced by the event generators GENIE [5] and HERWIG [6], where the latter is used only for the neutral current charm pair production. The resulting sample has been analyzed in order to look for processes with two visible particles decaying or mimicking a decay. This final selected sample has been used to train some classifier methods based on the TMVA software [7]. In Figure 5 can be seen the output variables for a Boost Decision Tree. The total amount of events expected with this topology is about 0.6 .

As can be seen from Figure 5, the dominant backgrounds corresponds to hadron re-interaction with the brick material. To check the reliability of the particle propagation, the same simulation software has been used to predict the results of the OPERA test beam [8]. Figure 6 presents the interaction lengths for pions obtained from the test beam and from the GEANT4 simulation.

Unfortunately, the capability to distinguish between pions and muons is not as good for the special event studied as it could have been, due to the fact track follow down procedure can not be performed on the tracks of this event. Therefore, at this stage, this analysis is underestimating the $v_{\mu}$ charged current contributions. Therefore, the value of the classifiers for this event is undefined, in order to avoid any bias. It will be evaluated only when the analysis will be satisfactorily completed.

\section{References}

[1] M. Guler et al. OPERA: An appearance experiment to search for $v_{\mu} \leftrightarrow v_{\tau}$ oscillations in the CNGS beam. Experimental proposal. (CERN-SPSC-2000-028, CERN-SPSC-P-318, LNGS-P25-00), 2000.

[2] N. Agafonova et al. Observation of tau neutrino appearance in the CNGS beam with the OPERA experiment. Progress of Theoretical and Experimental Physics, 2014(10), 2014.

[3] A. Kayis-Topaksu et al. Associated charm production in neutrino-nucleus interactions. Eur. Phys. J. C, 52(3):543-552, 2007.

[4] S. Agostinelli et al. GEANT4: A Simulation toolkit. Nucl.Instrum.Meth., A506:250-303, 2003.

[5] C. Andreopoulos et al. The GENIE Neutrino Monte Carlo Generator. Nucl. Instrum. Meth., A614:87-104, 2010.

[6] G. Corcella et al. Herwig 6: an event generator for hadron emission reactions with interfering gluons (including supersymmetric processes). Journal of High Energy Physics, 2001(01):010, 2001.

[7] A. Hoecker et al. TMVA: Toolkit for Multivariate Data Analysis. PoS, ACAT:040, 2007.

[8] H. Ishida et al. Study of hadron interactions in a lead-emulsion target. PTEP, 2014(9):093C01, 2014. 


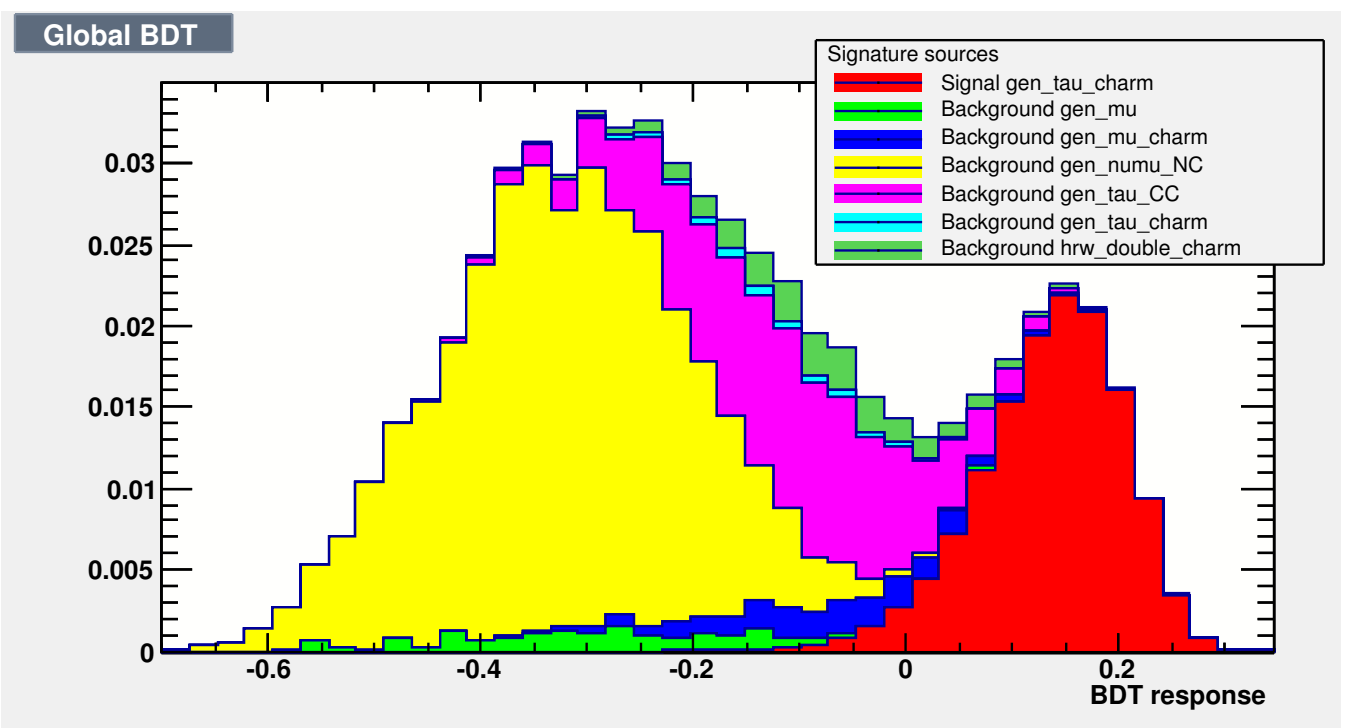

Figure 5: Example of a spectrum of the Boost Decision Tree output variable. This classifier has been specifically trained to look for a tau and charm sample (red). The main background is due to the NC interactions with 2 hadron re-interactions (yellow). In this plot, the contribution of $v_{\tau} \mathrm{CC}$ interaction is with one hadron re-interaction (magenta) is also considered as a background, although it would be a signal for the main OPERA purpose.

\section{Interaction Length}

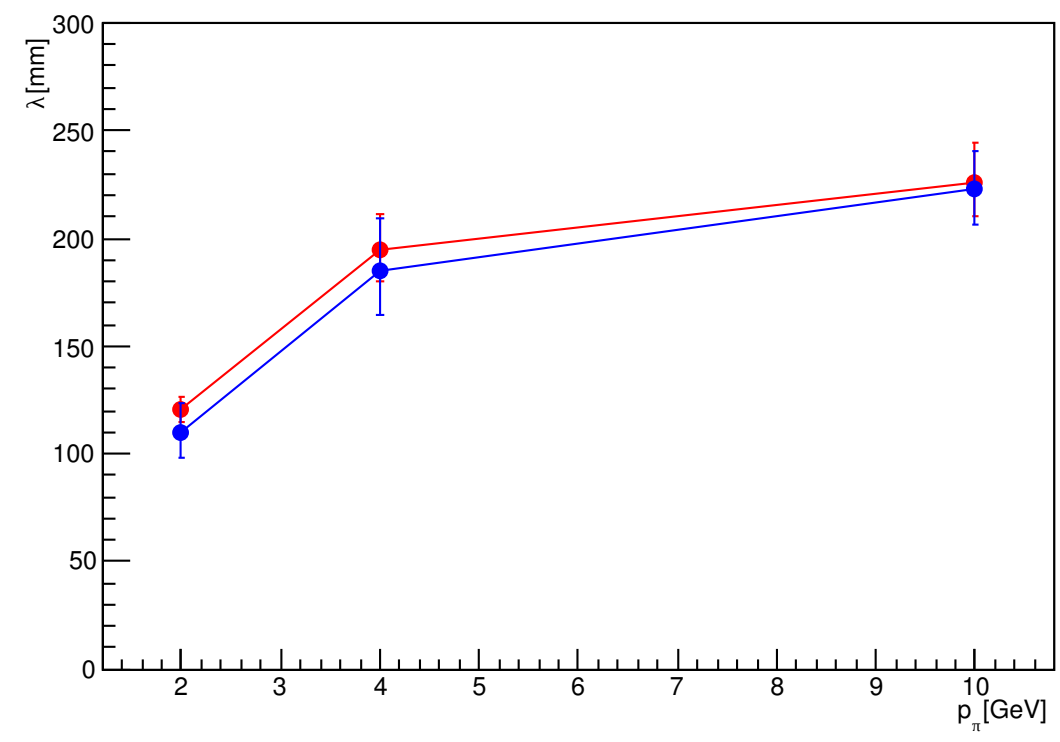

Figure 6: Comparison between the interaction length obtained from an OPERA test beam (blue) and the GEANT4 simulation (red). The interaction length is supposed to be almost constant as a function of the energy. The growth is due to the contribution of the multiple scattering inside the lead. In fact, at low energy the multiple scattering is larger and sometimes looks like an interaction with only one track coming out from the interaction vertex. This is accounted for in the simulation. 